\title{
Postencoding cognitive processes in the cross-race effect: Categorization and individuation during face recognition
}

\author{
Michael R. Ho ${ }^{1} \cdot$ Kathy Pezdek $^{1}$
}

Published online: 21 September 2015

(C) Psychonomic Society, Inc. 2015

\begin{abstract}
The cross-race effect (CRE) describes the finding that same-race faces are recognized more accurately than cross-race faces. According to social-cognitive theories of the CRE, processes of categorization and individuation at encoding account for differential recognition of same- and cross-race faces. Recent face memory research has suggested that similar but distinct categorization and individuation processes also occur postencoding, at recognition. Using a divided-attention paradigm, in Experiments $1 \mathrm{~A}$ and $1 \mathrm{~B}$ we tested and confirmed the hypothesis that distinct postencoding categorization and individuation processes occur during the recognition of same- and cross-race faces. Specifically, postencoding configural divided-attention tasks impaired recognition accuracy more for same-race than for cross-race faces; on the other hand, for White (but not Black) participants, postencoding featural divided-attention tasks impaired recognition accuracy more for cross-race than for same-race faces. A social categorization paradigm used in Experiments $2 \mathrm{~A}$ and $2 \mathrm{~B}$ tested the hypothesis that the postencoding ingroup or out-group social orientation to faces affects categorization and individuation processes during the recognition of same-race and cross-race faces. Postencoding out-group orientation to faces resulted in categorization for White but not for Black participants. This was evidenced by White participants' impaired recognition accuracy for same-race but not for cross-race out-group faces. Postencoding in-group orientation to faces had no effect on recognition accuracy for either samerace or cross-race faces. The results of Experiments 2A and
\end{abstract}

Michael R. Ho

Michael.Ho@cgu.edu

1 Department of Psychology, Claremont Graduate University, 150 East 10th Street, Claremont, CA 91711, USA
2B suggest that this social orientation facilitates White but not Black participants' individuation and categorization processes at recognition. Models of recognition memory for same-race and cross-race faces need to account for processing differences that occur at both encoding and recognition.

Keywords Cross-race effect · Own-race bias · Face recognition memory $\cdot$ Eyewitness memory

The cross-race effect (CRE) is a phenomenon in which individuals have better memory for faces of their own race than for faces of another race (Malpass \& Kravitz, 1969). A metaanalytic review by Meissner and Brigham (2001) reported that cross-race faces are 1.4 times more likely to be misidentified than same-race faces. The CRE is a robust effect that persists across various laboratory and real-world settings (Brigham, Bennett, Meissner, \& Mitchell, 2007) and has serious implications in the field of eyewitness memory (Fig. 1).

Various cognitive mechanisms have been proposed to account for the recognition disparity between same- and crossrace faces, including, for example, differential attention allocation (Levin, 2000) and social categorization (Sporer, 2001). Also, in terms of the dual-process framework, relatively more recollection of information occurs for same- than for crossrace faces (Meissner, Brigham, \& Butz, 2005). Furthermore, Hugenberg, Young, Bernstein, and Sacco (2010) proposed a categorization-individuation model (CIM) that consolidated previous cognitive models to account for differences in recognition accuracy for same- and cross-race faces. Although different cognitive mechanisms have been proposed to account for the CRE, these mechanisms have been hypothesized to occur exclusively at encoding (Young, Bernstein, \& Hugenberg, 2010). 

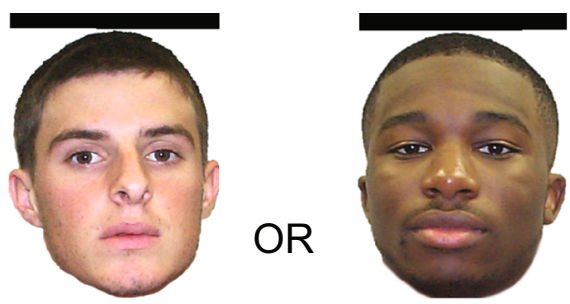

$+$

\begin{tabular}{ccclc}
\hline \multirow{2}{*}{$\begin{array}{c}\text { Control Divided } \\
\text { Attention }\end{array}$} & \multicolumn{2}{c}{ Configural Divided Attention } & \multicolumn{2}{l}{ Featural Divided Attention } \\
\cline { 2 - 5 } & Mouth & Eyes & Wide & Narrow \\
\hline
\end{tabular}
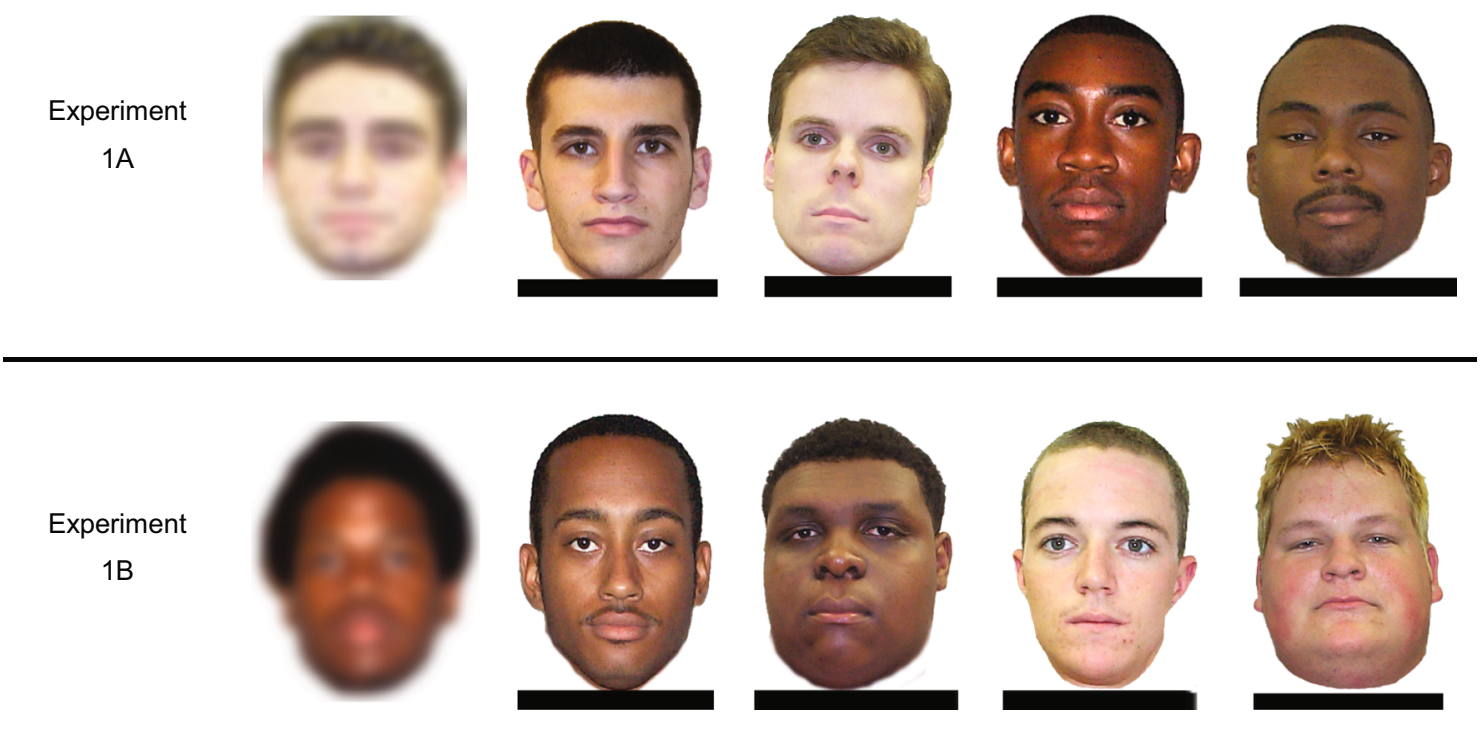

Fig. 1 Sample stimuli from Experiments 1A and 1B

According to the CIM, social categorization occurs at the first stage of face perception. Hugenberg et al. (2010) posited that the categorization of perceived faces as in-group (i.e., same-race) or out-group (i.e., cross-race) members signals whether perceivers will encode categorizing or individuating information. When a face is perceived as an out-group member, attention is directed to category-diagnostic information (e.g., hair or skin color) rather than to individuating information (e.g., the spatial configuration of facial features). This categorization or encoding of information salient to outgroup identity is referred to as featural processing (Farah, Tanaka, \& Drain, 1995). Conversely, faces perceived as ingroup members are individuated, meaning that distinctions in spatial and angular variations between similar but unique faces are attended to and encoded. This encoding process is referred to as configural processing (Maurer, Grand, \& Mondloch, 2002).
According to the CIM and related social-cognitive models (e.g., Sporer, 2001), successful identification generally yields greater social benefits for in-group than for out-group members, which motivates more individuation of same-race than of cross-race faces. However, although the CRE generally has been attributed to these processing differences at encoding, recent cognitive research has suggested that distinct processes involved in facial categorization and individuation occur at recognition, as well (Wammes \& Fernandes, 2015). In two experiments, we tested whether postencoding processing differences at recognition occur for same- and cross-race faces. Specifically, Experiments 1A (with White participants) and 1B (with Black participants) tested for postencoding configural and featural processes using a divided-attention paradigm. Then, Experiments 2A (with White participants) and $2 \mathrm{~B}$ (with Black participants) tested whether these postencoding configural and featural processes could be 
altered for same- and cross-race faces using a social categorization paradigm.

\section{Experiment 1A}

Performance is worse when two simultaneously performed tasks utilize similar rather than different cognitive processes (Fernandes \& Moscovitch, 2000). Applying this principle, Wammes and Fernandes (2015) tested and confirmed the hypothesis that the recognition of upright (i.e., configurally processed) and inverted (i.e., featurally processed) same-race faces is differentially impaired by the simultaneous completion of configural or featural judgment tasks at recognition. They reported that face recognition was less accurate when recognition and distractor tasks required simultaneous configural or simultaneous featural processing than when recognition and distractor tasks required dissimilar processing.

In Experiments 1A (with White participants) and 1B (with Black participants), we used simultaneous recognition and divided-attention tasks to test the hypothesis that categorization and individuation processes occur during the recognition of same- and cross-race faces, and not only at encoding. Specifically, in both experiments an interaction was predicted; recognition accuracy was predicted to be impaired more when participants completed the target and divided-attention tasks that required similar cognitive processes (i.e., same-race face recognition with configural divided-attention tasks or crossrace faces with featural divided-attention tasks), relative to when the target and divided-attention tasks required dissimilar cognitive processes (i.e., recognition of same-race faces with featural divided-attention tasks or cross-race faces with configural divided-attention tasks).

\section{Method}

\section{Participants and design}

Participants were recruited using Amazon's Mechanical Turk. The inclusion criteria required that participants be Caucasian non-Hispanic, reside in the United States, and score no more than three standard deviations from the mean (computed across all conditions) on the primary dependent variable, $d^{\prime}$. After removing three outliers, the final sample consisted of 153 participants $\left(M_{\text {age }}=38.34\right.$ years, $S D=13.00$; 93 female, 59 male, 1 did not specify). Following the methods proposed by Faul, Erdfelder, Lang, and Buchner (2007), the number of participants exceeded the sample size required to detect an effect size of $d=0.25$, alpha $=.05$, power $=.80$. This experiment was based on a 2 (Race of Target Face: same- or crossrace) $\times 3$ (Divided-Attention Task at Recognition: configural, featural, or control) between-subjects factorial design. Participants were randomly assigned to one of the six conditions; the number of participants in each condition is reported in Table 1.

\section{Materials and procedure}

The stimuli were obtained from a database of faces used by Meissner et al. (2005). ${ }^{1}$ In the presentation phase, participants viewed 15 same- or cross-race faces sequentially presented for $3 \mathrm{~s}$ each. Shortly afterward, during the test phase, they viewed 30 face pairs. Each pair was presented for $2 \mathrm{~s}$ and consisted of one face for a recognition task (indicated by a black bar above the face) and one face for a divided-attention task (indicated by a black bar below the face). After viewing each pair of test faces, participants were asked to make both a recognition and a divided-attention task judgment.

In the test phase, manipulations of the featural, configural, and control divided-attention tasks followed the methods used by Wammes and Fernandes (2015), with two exceptions: (a)In the featural divided-attention test condition, cross-race faces replaced inverted faces, and (b)these faces were selected on the basis of how wide open their eyes were rather than eye color. To create the featural divided-attention task at test, cross-race (i.e., Black) faces were selected such that half had wide eyes and half had narrow eyes. To create the configural divided-attention task at test, same-race (i.e., White) faces were slightly altered (by stretching or compressing the nose region) using GIMP 2, such that half of the faces had the nose closer to the eyes, and half had the nose closer to the mouth. Faces in the control divided-attention task at test were samerace (i.e., White) and blurred beyond recognition.

Recognition task The 30 recognition task faces ( 15 target and 15 foil) consisted of randomly presented same- or cross-race faces (corresponding to the race of faces viewed during presentation). Participants were instructed that in each pair of test faces, the face in the recognition task had a black bar above it, and they were to respond "old" or "new" to that face. Recognition task faces were counterbalanced so that each face approximately equally often served as a target and a foil face, and across face pairs, the recognition and divided-attention faces in each pair were randomly assigned to the left or right position.

Divided-attention task During the divided-attention task in the test phase, participants made featural, configural, or control divided-attention judgments (manipulated between subjects) while performing the recognition task. Participants were instructed to make these judgments on the face in each pair with a black bar below it. In the featural divided-attention task, participants viewed a cross-race face alongside each

\footnotetext{
${ }^{1}$ The photographs in our study were from a database maintained by Christian Meissner (http://iilab.utep.edu/stimuli.htm).
} 
Table 1 Mean $d^{\prime}$, hit rate (HR), false alarm rate (FAR), and $c$ values (with corresponding standard deviations), and $n$ per condition in Experiments 1A (White participants) and 1B (Black participants) (divided-attention task) and Experiments 2A (White participants) and 2B (Black participants) (social orientation task)

\begin{tabular}{|c|c|c|c|c|c|c|c|c|c|c|}
\hline \multirow[t]{2}{*}{ Condition } & \multicolumn{5}{|c|}{ Same-Race Target Faces } & \multicolumn{5}{|c|}{ Cross-Race Target Faces } \\
\hline & $d^{\prime}$ & HR & FAR & $c$ & $n$ & $d^{\prime}$ & HR & FAR & $c$ & $n$ \\
\hline \multicolumn{11}{|l|}{ Exp.1A } \\
\hline Featural & $1.33(1.04)$ & $.68(.20)$ & $.29(.19)$ & $.11(.55)$ & 30 & $0.38(0.75)$ & $.55(.20)$ & $.45(.22)$ & $.03(.61)$ & 24 \\
\hline Configural & $0.78(0.63)$ & $.61(.19)$ & $.34(.18)$ & $.09(.45)$ & 24 & $0.60(0.58)$ & $.62(.17)$ & $.42(.19)$ & $-.03(.60)$ & 21 \\
\hline Control & $1.25(0.83)$ & $.62(.22)$ & $.24(.20)$ & $.21(.68)$ & 26 & $0.92(0.68)$ & $.55(.18)$ & $.27(.21)$ & $.33(.56)$ & 28 \\
\hline \multicolumn{11}{|l|}{ Exp.1B } \\
\hline Featural & $0.80(0.71)$ & $.64(.18)$ & $.38(.20)$ & $-.01(.48)$ & 36 & $0.81(0.68)$ & $.57(.19)$ & $.32(.19)$ & $.19(.52)$ & 32 \\
\hline Configural & $0.15(0.74)$ & $.51(.21)$ & $.46(.20)$ & $.03(.45)$ & 33 & $0.53(0.66)$ & $.59(.24)$ & $.42(.22)$ & $-.03(.59)$ & 39 \\
\hline Control & $1.05(0.64)$ & $.60(.17)$ & $.26(.17)$ & $.24(.46)$ & 33 & $0.81(0.68)$ & $.60(.17)$ & $.32(.17)$ & $.14(.46)$ & 35 \\
\hline \multicolumn{11}{|l|}{ Exp.2A } \\
\hline In-group & $1.41(1.38)$ & $.67(.22)$ & $.32(.22)$ & $.05(.79)$ & 64 & $0.72(1.05)$ & $.59(.21)$ & $.39(.21)$ & $.03(.70)$ & 58 \\
\hline Out-group & $1.15(1.21)$ & $.63(.18)$ & $.32(.20)$ & $.17(.61)$ & 64 & $0.75(0.97)$ & $.56(.21)$ & $.33(.21)$ & .18 (.66) & 58 \\
\hline Control & $2.00(1.36)$ & $.64(.16)$ & $.21(.19)$ & $.41(.60)$ & 28 & $0.86(0.59)$ & $.58(.14)$ & $.34(.16)$ & $.12(.39)$ & 32 \\
\hline \multicolumn{11}{|l|}{ Exp.2B } \\
\hline In-group & $0.71(0.91)$ & $.55(.22)$ & $.33(.25)$ & $.26(.77)$ & 26 & $0.58(0.69)$ & $.58(.18)$ & $.40(.25)$ & $.07(.65)$ & 29 \\
\hline Out-group & $0.95(0.86)$ & $.56(.20)$ & $.30(.22)$ & $.26(.69)$ & 26 & $0.82(1.06)$ & $.59(.22)$ & $.36(.28)$ & $.12(.74)$ & 29 \\
\hline Control & $0.89(0.63)$ & $.57(.17)$ & $.28(.17)$ & $.26(.46)$ & 34 & $0.72(0.64)$ & $.60(.18)$ & $.36(.17)$ & $.06(.42)$ & 38 \\
\hline
\end{tabular}

The two control groups in Experiments 2A and 2B were "floating" comparison groups; the in-group and out-group conditions were manipulated within subjects

target or foil face and responded whether this face had eyes that were "wide" or "narrow." In the configural dividedattention task, participants viewed a same-race face alongside each target or foil face and responded whether this face had a nose that was closer to the "eyes" or "mouth." In the control divided-attention task, participants viewed a blurred face alongside each target or foil face and were instructed to disregard this face.

\section{Results and discussion}

Recognition memory performance was analyzed in terms of the primary variable $d^{\prime}$, as well as hit rate, false alarm rate, and $c$ (criterion response) data. Following methods proposed by Macmillan and Creelman (2005), hit and false alarm rates equal to 0 were converted to $1 /(2 N)$, and hit rates and false alarms equal to 1 were converted to $1-1 /(2 N)$. The mean $d^{\prime}$, hit rate, false alarm rate, and criterion values (with standard deviations) for each condition are presented in Table 1.

A 2 (Race of Target Face: same- or cross-race) $\times 3$ (Divided-Attention Task at Recognition: configural, featural, or control) analysis of variance (ANOVA) was conducted on each of the four dependent measures separately. Our major hypothesis was confirmed in the analysis of $d^{\prime}$ data: The interaction between face race and divided-attention task was significant, $F(2,147)=3.48, p=.033, \eta^{2}=.05$. As can be seen in Table 1 , and as was confirmed by simple effects tests, the recognition of same-race faces was less accurate in the configural than in the control divided-attention task condition, $t(48)=2.26$, $p=.028$; however, recognition accuracy for same-race faces did not differ between the control and featural dividedattention task conditions, $t(54)=0.32, p=.75$. Conversely, recognition of cross-race faces was less accurate in the featural than in the control divided-attention task condition, $t(50)=$ $2.70, p=.009$; however, the difference in recognition accuracy for cross-race faces in the control and configural dividedattention task conditions was nonsignificant, $t(47)=1.71$, $p=.095$. In addition, and confirming the CRE, recognition was more accurate for same-race $(M=1.14, S D=0.89)$ than for cross-race $(M=0.65, S D=0.71)$ faces, $F(1,147)=14.58$, $p<.001, \eta^{2}=.09$. The main effect of divided-attention task was also significant, $F(2,147)=3.17, p=.045, \eta^{2}=.04$; recognition was significantly less accurate in the configural $(M=0.70, S D=0.61)$ than in the full-attention $(M=1.07$, $S D=0.77$ ) condition, $t(97)=2.71, p=.008$; no other pairwise differences among conditions were significant.

In the analysis of the hit rate data, only the main effect of face race was marginally significant, $F(1,147)=3.79, p=$ $.054, \eta^{2}=.03$; mean hit rates were higher for same-race $(M=$ $.64, S D=.20)$ than for cross-race $(M=.57, S D=.18)$ faces. In the analysis of the false alarm rate data, false alarm rates were higher for cross-race $(M=.37, S D=.22)$ than for same-race $(M=.29, S D=.19)$ foil faces, $F(1,147)=8.02, p=.005, \eta^{2}=$ .05 . We also found a main effect of divided-attention task at 
recognition, $F(2,147)=6.44, p=.002, \eta^{2}=.08$. Simple effects tests indicated that false alarm rates were significantly higher in the featural $(M=.36, S D=.21)$ than in the control $(M=.26$, $S D=.20)$ divided-attention task, $t(106)=2.62, p=.01$, and significantly higher in the configural $(M=.38, S D=.19)$ than in the control $(M=.26, S D=.20)$ divided-attention task, $t(106)=3.09, p=.003$. The interaction was not significant in the analysis of false alarm rate data. In the analysis of $c$, no significant effects were visible.

The principal finding in Experiment1A was the significant interaction effect of the race of a target face and the dividedattention task at recognition on $d^{\prime}$ data. As we predicted, postencoding configural divided-attention tasks impaired recognition accuracy more for same- than for cross-race faces. Postencoding featural divided-attention tasks impaired recognition accuracy more for cross- than for same-race faces. Critically, these results extend current CRE models such as the $\mathrm{CIM}$ and suggest that categorization and individuation processes occur postencoding as well as at encoding.

\section{Experiment 1B}

Experiment1B tested whether the results of Experiment $1 \mathrm{~A}$ with White participants generalize to Black participants. Two meta-analyses had reported the magnitude of the CRE to be larger for White than for Black participants (Anthony, Copper, \& Mullen, 1992; Meissner \& Brigham, 2001), whereas a third (Bothwell, Brigham, \& Malpass, 1989) had reported no statistically significant difference between the magnitudes of the CRE for White versus Black participants. Anthony et al. suggested that relatively more individuating (i.e., configural) than categorizing (i.e., featural) information is encoded when individuals view out-group faces from majority groups and that this attenuates the CRE. Although the CRE does not appear to be as large and consistent for Black as for White participants, it is important to test whether these differences in the CRE are reflected in postencoding processes by Black participants. Experiment1B was a replication of Experiment $1 \mathrm{~A}$ with Black participants. If outgroup size (i.e., majority status) does not impact the recognition of same- and cross-race faces, recognition accuracy for Black participants would be predicted to mirror the pattern of results produced by White participants in Experiment $1 \mathrm{~A}$. On the other hand, if the majority status of an out-group increases the amount of configural information processed for cross-race faces (Anthony et al. 1992), recognition accuracy would be predicted to be impaired more in the configural divided-attention condition than in the full and featural divided-attention conditions for both same- and cross-race faces. However, within the configural divided-attention condition, it would be predicted that cross-race faces would be recognized better than same-race faces.

\section{Method}

Participants and design The recruitment of Black participants in Experiment1B followed the procedures used in Experiment $1 \mathrm{~A}$. After removing six outliers, the final sample consisted of 208 participants $\left(M_{\mathrm{age}}=31.88\right.$ years, $S D=8.96$; 99 female, 105 male, 4 did not specify). Like Experiment 1A, Experiment 1B had a 2 (Race of Target Face: same- or crossrace) $\times 3$ (Divided-Attention Task at Recognition: configural, featural, or control) between-subjects factorial design.

Materials and procedure The Experiment1B stimuli were the same as those used in Experiment1A. Similarly, the presentation and test phases in Experiment1B were identical to those in Experiment1A, with three exceptions. First, to create the featural divided-attention task at test, cross-race (i.e., White) faces were selected, such that half had wide eyes and half had narrow eyes. Second, to create the configural dividedattention task at test, same-race (i.e., Black) faces were slightly altered (by stretching or compressing the nose region) using GIMP 2, such that half of the faces had the nose closer to the eyes, and half had the nose closer to the mouth. Third, the faces in the control divided-attention task at test were samerace (i.e., Black) and blurred beyond recognition.

\section{Results and discussion}

Recognition memory performance was analyzed following the procedures used in Experiment $1 \mathrm{~A}$. The mean $d^{\prime}$, hit rate, false alarm rate, and $c$ (criterion response) values (with standard deviations) for each condition are reported in Table 1.

A 2 (Race of Target Face: same- or cross-race) $\times 3$ (Divided-Attention Task at Recognition: configural, featural, or control) ANOVA was conducted on each of the four dependent measures separately. First, in the analysis of $d^{\prime}$ data, consistent with results reported elsewhere, recognition accuracy for same-race $(M=0.67$, $S D=0.79)$ and cross-race $(M=0.72, S D=0.67)$ faces did not differ significantly, $F(1,202)=0.42, p=.52$, $\eta^{2}=.002$; there was not a significant CRE for our Black participants.

Our major hypothesis was tested with the interaction between face race and divided-attention task; this interaction was significant, $F(2,202)=3.40, p=.035, \eta^{2}=$ .03 . Consistent with the results of White participants in Experiment1A, the recognition of same-race faces was less accurate in the configural than in the control divided-attention task condition, $t(64)=5.30, p<.001$, and the difference in recognition accuracy for samerace faces in the control and featural divided-attention 
task conditions did not differ significantly, $t(67)=1.50$, $p=.14$. However, and inconsistent with the results of Experiment1A, for Black participants, the recognition of cross-race faces was less accurate in the configural than in the control divided-attention task condition, $t(72)=$ $2.00, p=.049$, and the difference in recognition accuracy for cross-race faces in the control and featural divided-attention task conditions was nonsignificant, $t(65)=0.14, p=.89$. Interestingly, recognition accuracy in the configural divided-attention task was lower for same- than for cross-race faces, $t(70)=2.32, p=.023$. The main effect of divided-attention task was significant, $F(2,202)=15.18, p<.001, \eta^{2}=.13$; recognition was significantly less accurate in the configural $(M=$ $0.35, S D=0.72)$ than in the full-attention $(M=0.94$, $S D=0.65)$ condition, $t(138)=5.04, p<.001$; recognition was also significantly less accurate in the configural $(M=0.35, S D=0.72)$ than in the featural $(M=0.81$, $S D=0.69)$ divided-attention condition, $t(138)=3.80, p$ $<.001$.

In the analysis of the hit rate data, no significant results emerged. In the analysis of the false alarm rate data, a main effect of divided-attention task at recognition was observed, $F(2,202)=11.30, p<.001, \eta^{2}=.10$. Simple effects tests indicated that false alarm rates were significantly higher in the configural $(M=.44, S D=.21)$ than in the control $(M=.29, S D=.17)$ divided-attention task, $t(138)=4.57, p<.001$, and significantly higher in the configural $(M=.44, S D=.21)$ than in the featural $(M=.35, S D=.19)$ divided-attention task, $t(138)=3.09$, $p=.007$. Neither the main effect of face race nor the interaction was significant in the analysis of false alarm rate data. In the analysis of criterion, $c$, we found no significant results.

The results of Experiment1B differed in important ways from those of Experiment1A. First, consistent with results reported elsewhere, the CRE was not significant for Black participants in Experiment1B. Furthermore, the significant interaction between race of target face and divided-attention task at recognition on $d^{\prime}$ was significant in Experiment1B; however, unlike in Experiment1A, the interaction in Experiment1B resulted from the configural divided-attention task impairing recognition more for same- than for cross-race faces. The results from Experiment1B-specifically, the finding that postencoding configural divided-attention tasks impaired recognition for both same- and cross-race facessupport the findings of Anthony et al. (1992) and suggest that more configural than featural information is recognized when individuals view cross-race faces that are from majority groups. The absence of the CRE in Experiment1B can be accounted for by this relative increase in the configural processing of cross-race faces.
More critically, and consistent with Experiment1A, in Experiment 1B postencoding processes impaired the recognition of both same- and cross-race faces.

\section{Experiment 2A}

According to the CIM, when motivation to individuate increases, as it typically does for in-group members, attention is directed to encoding configural information. When motivation to individuate decreases, as it typically does for out-group members, attention is directed to encoding featural information. These encoding differences result in less accurate recognition for out-group than for in-group faces. Manipulations of social labels (Bernstein, Young, \& Hugenberg, 2007) at encoding have also been shown to impact perceivers' motivation to encode individuating information. Hehman, Mania, and Gaertner (2010) reported that altering in-group/out-group distinctions at encoding mitigates the CRE by increasing recognition for in-group-labeled cross-race faces or decreasing recognition for out-group-labeled same-race faces.

Experiments $1 \mathrm{~A}$ and $1 \mathrm{~B}$ provided evidence for the presence of postencoding individuation and categorization processes during the recognition of same- and cross-race faces. These results suggest that manipulating the in-group or outgroup social orientation of same- or cross-race faces postencoding may also impact recognition in a pattern that mirrors processing at encoding: Postencoding of in-group labels should direct attention to the recognition of configural information, and out-group labels should direct attention to the recognition of featural information.

Although social orientation at test was predicted to direct participants' attention to configural (i.e., on in-group faces) or featural (i.e., on out-group faces) information, it was also predicted that the impact of social orientation at test would be limited by the cognitive processes that occurred at encoding. For example, if participants encoded featural information from presented faces, this should limit their recognition of configural information on these faces at test, regardless of whether or not attention at test was directed to configural information. Experiments 2A (with White participants) and 2B (with Black participants) utilized the social categorization task used by Bernstein et al. (2007) to investigate the impact of postencoding social orientation on the recognition of sameand cross-race faces. If postencoding out-group social orientation directs attention to the recognition of featural information, then out-group social orientation should impair recognition accuracy more for same- than for cross-race faces, relative to faces with no social orientation. If postencoding in-group social orientation directs attention to the recognition of configural information, then in-group social orientation should have little impact on recognition accuracy for sameor cross-race faces, relative to faces with no social orientation. 


\section{Method}

Participants and design In Experiments 1A and 2A, we used identical recruitment, inclusion criteria, and power analyses for the required sample size. After removing one outlier, the final sample in Experiment2A was 182 participants $\left(M_{\text {age }}=\right.$ 40.01 years, $S D=13.30$; 93 female, 87 male, 2 did not specify). This experiment had a 2 (Race of Target Face: same- or cross-race) $\times 2$ (Social Orientation: in-group, out-group) mixed-factorial design, with Face Race as the betweensubjects factor. Two "floating" control conditions were included, as well; two groups of participants were assigned to either a no-social-orientation condition viewing same-race faces or a no-social-orientation condition viewing cross-race faces. These two control groups served as comparison groups but were not part of the $2 \times 2$ mixed-factor design in which social orientation condition was manipulated within subjects. ${ }^{2}$

Materials and procedure The face stimuli in Experiments 1 and 2 were identical, with two exceptions: The faces used in Experiment2A (a)had no black bars above or below them and (b) were manipulated using GIMP 2, such that the white space surrounding the faces had green or red backgrounds.

Experiment2A consisted of three phases: presentation, personality assignment, and test. In the presentation phase, participants viewed 15 same- or cross-race faces presented sequentially for $3 \mathrm{~s}$ each. In the personality assignment phase, participants completed a faux personality test following procedures used by Young et al. (2010). After this test, each participant was randomly assigned as a red, green, or $N$ personality and provided with feedback regarding the characteristics of her/his personality type; in fact, identical personality descriptions were provided to every participant.

In the test phase, participants viewed test faces each with a red or green background and were told that the background color indicated the personality type of each person viewed. Thereby, test faces with background colors that matched each participant's faux personality types were socially oriented to as in-group members at recognition; test faces with background colors that did not match each participant's personality type were socially oriented to as out-group members at recognition. Participants in the control conditions were assigned to the $N$ personality; they were told that the background color had no meaning and could be ignored. During the test phase, participants viewed 30 randomly arranged same- or cross-race

\footnotetext{
${ }^{2}$ After our participants were randomly assigned faux personalities, they viewed faces with colored backgrounds to increase the social salience of the target faces. However, in the no-social-orientation control conditions, the background colors of target faces had no meaning. As such, the two separate control groups served as "floating" comparison groups but were not part of the $2 \times 2$ mixed-factor design in which social orientation condition was manipulated within subjects.
}

faces ( 15 targets and 15 foils) and responded "old" or "new" to each. Background colors and the assignment of each face to the target or foil condition were counterbalanced across conditions.

\section{Results and discussion}

As in Experiments $1 \mathrm{~A}$ and $1 \mathrm{~B}$, recognition memory performance was analyzed in terms of the primary variable $d^{\prime}$, as well as hit rate, false alarm rate, and criterion (c) data. The means (with standard deviations) for each dependent variable and each condition are reported in Table 1. First, a 2 (Race of Target Face: same- or cross-race) $\times 2$ (Social Orientation: ingroup or out-group) ANOVA was conducted on each of the four dependent measures separately. In the analysis of $d^{\prime}$ data, only the main effect of face race was significant, $F(1,120)=$ $11.58, p=.01, \eta^{2}=.09$; reflecting the CRE, same-race faces $(M=1.28, S D=0.89)$ were more accurately recognized than cross-race faces $(M=0.74, S D=0.88)$. In the analysis of the hit rate data, only the main effect of face race was significant; hit rates were higher for same-race $(M=.65, S D=.17)$ than for cross-race $(M=.58, S D=.17)$ faces, $F(1,120)=5.36, p=.022$, $\eta^{2}=.04$. In the analysis of the false alarm rate data, no significant effects resulted. In the analysis of $c$, only the main effect of social orientation was significant, with participants being more liberal saying "Yes" ("I have seen this face before") when viewing out-group faces $(M=.17, S D=.63)$ than when viewing in-group faces $(M=.05, S D=.79), F(1,120)=5.36$, $p=.039, \eta^{2}=.04$.

More central to the hypotheses in Experiment2A, a second set of analyses was conducted to assess whether recognition accuracy in either of the two social orientation conditions differed from that in the control condition. Because this was a floating control condition, it was necessary to conduct four $t$ tests on the primary dependent variable, $d^{\prime}$ (see note 2 ). These contrasts tested the hypothesis that, relative to the control group, socially orienting target faces to be out-group members at recognition would decrease recognition accuracy for samebut not for cross-race faces. These results can be seen in Table 1. As predicted, same-race faces in the out-group social orientation condition were recognized less accurately than same-race faces in the control condition, $t(90)=2.98$, $p=.004, d=-0.66$; the difference in recognition accuracy between same-race faces in the control and in-group social orientation conditions was nonsignificant. Furthermore, as predicted, for cross-race faces, no significant differences resulted between the control group and either the in-group or the out-group social orientation condition.

The primary finding in Experiment2A was that, relative to the same-race control condition, recognition accuracy decreased in the same-race out-group but not in-group social orientation condition; however, relative to the cross-race control condition, no effects of social orientation on the 
recognition of cross-race faces were observed. These results support the hypotheses that at test, out-group social orientation directs attention to the recognition of featural facial information, and in-group social orientation directs attention to the recognition of configural facial information. More broadly, the findings from Experiment 2 suggest that manipulating the perceiver's motivation to individuate at recognition mirrors the effects of configural and featural processes observed at encoding in other studies.

\section{Experiment 2B}

In Experiment2B, we tested whether the results of Experiment2A with White participants generalize to Black participants. Experiment2B followed the logic of Experiment2A to test whether postencoding in-group labels would direct attention to the recognition of configural information, and whether out-group labels would direct attention to the recognition of featural information.

\section{Method}

Participants and design The inclusion criteria, recruitment methods, and power analyses for sample size were identical in Experiments $2 \mathrm{~A}$ and $2 \mathrm{~B}$, with the exception that the participants in Experiment2B were Black. After removing six outliers, the final sample in Experiment2B was 127 participants $\left(M_{\text {age }}=31.72\right.$ years, $S D=9.16 ; 77$ female, 50 male). Like Experiment2A, this experiment had a 2 (Race of Target Face: same- or cross-race) $\times 2$ (Social Orientation: in-group, outgroup) mixed-factorial design with Face Race as the between-subjects factor. Similarly, this experiment had two "floating" control groups that served as comparison groups but were not part of the $2 \times 2$ mixed-factor design.

Materials and procedure The face stimuli and procedures in Experiment2B were identical to those used in Experiment2A.

\section{Results and discussion}

Recognition memory performance was analyzed in terms of the primary dependent variable $d^{\prime}$, as well as hit rate, false alarm rate, and criterion (c) data. The means (with standard deviations) for each dependent variable and each condition are reported in Table 1. First, a 2 (Race of Target Face: same- or cross-race) $\times 2$ (Social Orientation: in-group or out-group) ANOVA was conducted on each of the four dependent measures separately. In the analyses of the $d^{\prime}$, hit rate, false alarm rate, and $c$ data, we found no significant effects (all $F_{\mathbf{S}}<3.16$ ). Critically, in the analysis of $d^{\prime}$ data, consistent with the results reported elsewhere, including Experiment 1B, recognition accuracy for same-race $(M=0.83, S D=1.06)$ and cross-race $(M=$
$0.70, S D=1.00)$ faces did not differ significantly for Black participants, $F(1,53)=0.44, p=.51, \eta^{2}=.008$. Similarly, no significant effects emerged in the analyses that assessed whether recognition accuracy in either of the two social orientation conditions differed from that in the "floating" control condition.

The primary finding from Experiment2B was that postencoding social orientation had no significant impact on recognition accuracy for Black participants. The CRE literature has reported that categorization and individuation encoding processes in the CRE are weak or inconsistent for Black participants. Given that the CRE was not replicated with Black participants in either Experiment1B or 2B, our more specific findings in these two experiments should be interpreted cautiously.

\section{General discussion}

The cross-race effect has been investigated extensively over the past decades. Hugenberg et al. (2010) posited, "One commonality of the CIM with most other theories of the $\mathrm{ORE}^{3}$ is the shared focus on the encoding phase, rather than storage or recognition phases" (p.1183). Contrary to this view, recent research has suggested that distinct cognitive processes also occur postencoding during facial recognition (Wammes \& Fernandes, 2015). In our study, we investigated the cognitive mechanisms specifically underlying the CRE that operate at recognition.

In Experiment 1A, an interference paradigm tested and confirmed the hypothesis that categorization and individuation processes occur during the recognition of same- and cross-race faces for White participants. In Experiment2A, a social categorization paradigm tested and confirmed the hypothesis that manipulating perceiver motivation to individuate at recognition differentially impacts recognition accuracy for same- and cross-race faces for White participants. Specifically, a postencoding out-group social orientation task directed attention to the recognition of featural information on target faces; a postencoding in-group social orientation task directed attention to the recognition of configural information on target faces.

Three novel findings from this study extend the literature on the CRE, specifically for White participants. First, distinct postencoding cognitive processes (i.e., individuation and categorization) occur during the recognition of same- and cross-race faces. Second, these postencoding processes mirror the encoding processes reported elsewhere; typically, same-race faces are configurally encoded and recognized, and cross-race

\footnotetext{
${ }^{3}$ Own-race effect
} 
faces are featurally encoded and recognized. Third, using a postencoding social orientation manipulation, these processes vary with perceivers' increased or decreased motivation to individuate faces at recognition.

Experiments $1 \mathrm{~B}$ and $2 \mathrm{~B}$ tested the generalization of the findings with White participants to Black participants, for whom the cross-race (White) faces were a majority race group; the generalization was limited. In Experiment1B, an interference paradigm tested and confirmed the hypothesis that categorization processes occur during the recognition of same- and cross-race faces for Black participants. However, contrary to the results of Experiment $1 \mathrm{~A}$, postencoding featural divided-attention tasks did not interfere with Black participants' recognition of cross-race faces. And, contrary to the findings in Experiment2A, in Experiment2B, with the social categorization paradigm, we found no significant differences in recognition accuracy between same- and crossrace faces; when Black participants' motivation to individuate was manipulated at recognition, no changes in recognition accuracy were observed. This difference in face recognition patterns observed between White and Black participants is unsurprising, given that the CRE was not observed among Black participants in Experiments 1B and 2B. Additional research will be needed to clarify differences in postencoding recognition processes between Black and White participants.

Although these results enhance theoretical models of the CRE, it is also worth noting the practical implications of this research. This study suggests that the contextual factors or methods implemented during the recognition of same- and cross-race faces (e.g., during eyewitness identification procedures) impair recognition accuracy. Specifically, the different cognitive processing at encoding and recognition (i.e., individuation at encoding and categorization at recognition) is likely to reduce recognition accuracy for both same- and cross-race faces. For example, Ackerman et al. (2006) reported that angry facial expressions improved recognition for cross-race but not for same-race faces, and they suggested that perceived threat increased motivation to individuate cross-races faces at encoding. Similarly, witnessing a crime may increase bystander motivation to encode individuating information. However, eyewitnesses may view police line-ups in which suspects are oriented to as out-group members (e.g., cross-race, criminal, or low socio-economic status), thus invoking categorization processes at recognition. Motivating participants to individuate at recognition may mitigate this processing disparity, by directing attention to the recognition of configural information that was previously encoded.

Given the novel nature of our findings and the important applied implications, further research on postencoding motivation (e.g., instructions, emotion of target faces, and social orientation) and categorization/ individuation processes is warranted. Despite the CRE being studied primarily as an encoding phenomenon, it is clear from our findings that postencoding cognitive processes also impact the recognition of same- and cross-race faces.

\section{References}

Ackerman, J. M., Shapiro, J. R., Neuberg, S. L., Kenrick, D. T., Becker, D. V., Griskevicius, V., \& Schaller, M. (2006). They all look the same to me (unless they're angry): From out-group homogeneity to out-group heterogeneity. Psychological Science, 17, 836-840. doi:10.1111/j.1467-9280.2006.01790.x

Anthony, T., Copper, C., \& Mullen, B. (1992). Cross-racial facial identification: A social cognitive integration. Personality and Social Psychology Bulletin, 18, 296-301. doi:10.1177/0146167292183005

Bernstein, M. J., Young, S. G., \& Hugenberg, K. (2007). The crosscategory effect: Mere social categorization is sufficient to elicit an own-group bias in face recognition. Psychological Science, 18, 706712. doi:10.1111/j.1467-9280.2007.01964.x

Bothwell, R. K., Brigham, J. C., \& Malpass, R. S. (1989). Cross-racial identification. Personality and Social Psychology Bulletin, 15, 19 25. doi: $10.1177 / 0146167289151002$

Brigham, J. C., Bennett, L. B., Meissner, C. A., \& Mitchell, T. (2007). The influence of race on eyewitness memory. In M. P. Toglia (Ed.), The handbook of eyewitness psychology: Memory for people (pp. 257-281). Mahwah, NJ: Erlbaum.

Farah, M. J., Tanaka, J. W., \& Drain, H. M. (1995). What causes the face inversion effect? Journal of Experimental Psychology. Human Perception and Performance, 21, 628-634. doi:10.1037/00961523.21.3.628

Faul, F., Erdfelder, E., Lang, A. G., \& Buchner, A. (2007). G*Power 3: A flexible statistical power analysis program for the social, behavioral, and biomedical sciences. Behavior Research Methods, 3, 175-191. doi:10.3758/BF03193146

Fernandes, M. A., \& Moscovitch, M. (2000). Divided attention and memory: Evidence of substantial interference effects at retrieval and encoding. Journal of Experimental Psychology: General, 129, 155-176. doi:10.1037/0096-3445.129.2.155

Hehman, E., Mania, E. W., \& Gaertner, S. L. (2010). Where the division lies: Common in-group identity moderates the cross-race-facial recognition effect. Journal of Experimental Social Psychology, 46, 445-448. doi:10.1016/j.jesp.2009.11.008

Hugenberg, K., Young, S. G., Bernstein, M. J., \& Sacco, D. F. (2010). The categorization-individuation model: An integrative account of the other-race recognition deficit. Psychological Review, 117, 11681187. doi: $10.1037 / \mathrm{a} 0020463$

Levin, D. T. (2000). Race as a visual feature: Using visual search and perceptual discrimination tasks to understand face categories and the cross-race recognition deficit. Journal of Experimental Psychology: General, 129, 559-574. doi:10.1037/0096-3445.129.4.559

Macmillan, N. A., \& Creelman, C. D. (2005). Detection theory: A user's guide (2nd ed.). Mahwah, NJ: Erlbaum.

Malpass, R. S., \& Kravitz, J. (1969). Recognition for faces of own and other race. Journal of Personality and Social Psychology, 13, 330 334. doi: $10.1037 / \mathrm{h} 0028434$

Maurer, D., Grand, R. L., \& Mondloch, C. J. (2002). The many faces of configural processing. Trends in Cognitive Sciences, 6, 255-260. doi:10.1016/s1364-6613(02)01903-4 
Meissner, C. A., \& Brigham, J. C. (2001). Thirty years of investigating the own-race bias in memory for faces: A meta-analytic review. Psychology, Public Policy, and Law, 7, 3-35. doi:10. 1037/1076-8971.7.1.3

Meissner, C. A., Brigham, J. C., \& Butz, D. A. (2005). Memory for ownand other-race faces: A dual-process approach. Applied Cognitive Psychology, 19, 545-567. doi:10.1002/acp.1097

Sporer, S. L. (2001). Recognizing faces of other ethnic groups: An integration of theories. Psychology, Public Policy, and Law, 7, 36-97. doi:10.1037/1076-8971.7.1.36
Wammes, J. D., \& Fernandes, M. A. (2015). Interfering with memory for faces: The cost of doing two things at once. Memory. doi:10.1080/ 09658211.2014.998240

Young, S. G., Bernstein, M. J., \& Hugenberg, K. (2010). When do own-group biases in face recognition occur? Encoding versus post-encoding. Social Cognition, 28, 240-250. doi:10.1521/ soco.2010.28.2.240 